\title{
Templated self-assembly of wedge-shaped DNA arrays
}

Daniel Lubrichª, , Jonathan Bath ${ }^{\mathrm{a}}$ and Andrew J. Turberfielda, ${ }^{\mathrm{a}}$

aUniversity of Oxford, Department of Physics, Clarendon Laboratory, Parks Road, Oxford OX1 3PU, UK

bDepartment of Physics, National University of Singapore, 117542 Singapore, Republic of Singapore

*Corresponding author. e-mail: a.turberfield@physics.ox.ac.uk.

\begin{abstract}
We demonstrate the use of a one-dimensional template to control the shape of a twodimensional array self-assembled from a minimal set of DNA tiles. A periodic single-stranded template seeds tile assembly. A unique vertex tile at the 5' end of the template controls the positioning of edge and body tiles to create a wedge-shaped array. The vertex angle of the array is approximately $12^{\circ}$; edge lengths are of the order of $1 \mu \mathrm{m}$.
\end{abstract}

Keywords self-assembly, DNA nanotechnology, supramolecular chemistry, algorithmic assembly

\section{Introduction}

Nanofabrication by DNA self-assembly exploits the specificity of Watson-Crick base-pairing to create intricate molecular structures from synthetic oligonucleotides. ${ }^{1}$ Static DNA structures can be used to explore strategies for the control of the self-assembly process and provide the basis for the development of active molecular devices, including rudimentary molecular machines. ${ }^{2}$ Twodimensional DNA arrays, ${ }^{3-6}$ characterized by the repeated use of a small number of building blocks ('tiles'), are attractive because large structures (up to $1 \mathrm{~mm})^{7}$ can be fabricated from a relatively small number of DNA strands (oligonucleotides). Each tile consists of a small number of strands held together by hybridization: interactions between tiles are by hybridization of single-stranded 'sticky ends' (typically four to a tile). The structure of the array is determined by the pattern of complementarity between the sticky ends presented by the set of tiles. Extended arrays are 
usually made by mixing components (either individual strands or pre-formed tiles) and cooling slowly through the temperature range in which assembly occurs.

A number of strategies have been developed to control the assembly of extended structures from DNA to create defined shapes. The use of a unique tile for each site of an assembly is the simplest way to control size and shape, ${ }^{8}$ but has the drawback that the required number of components scales with the square of the linear dimension. The system size can be reduced by using the same tile type in symmetry-related sites $^{9}$ and by using multiple assembly steps. ${ }^{10,11}$ Algorithmic selfassembly is a flexible strategy for the construction of complex patterns and shapes from few components: row-by-row assembly of a 2D array is mapped onto the operation of a 1D cellular automaton ${ }^{12}$ such that successive rows of tiles correspond to successive states of the automaton. Each new tile binds to two tiles in the previous row by hybridization to one sticky end from each, and displays two sticky ends which are used to bind tiles in the next row. The initial row of tiles corresponds to the program of the automaton, and the pattern of sticky ends on the set of assembling tiles embodies its rule table. Algorithmic assembly has been used to assemble complex tile patterns; ${ }^{13-15}$ the size of the tile set required to create a given array shape has been analyzed. ${ }^{16}$ An array border may be constructed from discrete edge tiles ${ }^{17}$ or as an extended seed structure ${ }^{13,18,19}$ which may be assembled on a long DNA template strand. ${ }^{13,18}$

Here we report the use of a template strand in combination with special edge tiles to control the shape of DNA arrays. The arrays are $2 \mathrm{~nm}$ wide at the vertex with an angle of $12 \pm 2^{\circ}$ and edge lengths up to $1 \mathrm{\mu m}$. The largely periodic template strand defines one edge of the array. The template strand is made by rolling circle replication, ${ }^{20-22}$ which allows incorporation of an unique sequence of bases at its 5' end: this motif is used to initiate assembly of edge tiles to form the other side of the wedge. The body of the array is formed from a single type of tile. The template seeds array formation: in the designed assembly sequence, each new tile added to the growing array is held by cooperative binding of two sticky ends. Body tiles on their own can form arrays with uncontrolled size and shape, but this off-template assembly is suppressed by an activation 
barrier corresponding to the formation of a crystallization nucleus ${ }^{19}$ The template thus determines array shape in a non-equilibrium assembly process.

\section{System Design}

A single-stranded DNA with a repeating sequence motif can be made by rolling circle replication of

a (-) sense single-stranded circular substrate using the processive phi29 polymerase. ${ }^{23}$ The rolling circle replication product can serve as a template for assembly of a one-dimensional array of DNA tiles. ${ }^{24-26}$ A unique motif can be incorporated at the 5' end of the template strand simply by appending it to the $5^{\prime}$ end of the replication primer (Figure 1A): this allows the positioning of a unique tile at the $5^{\prime}$ end of the template. Here we use a one-dimensional array of tiles formed directly on the template to seed the cooperative growth of a two-dimensional array and a unique tile at the $5^{\prime}$ end of the template to determine the shape of the array.

\section{A Rolling circle replication and template strand}

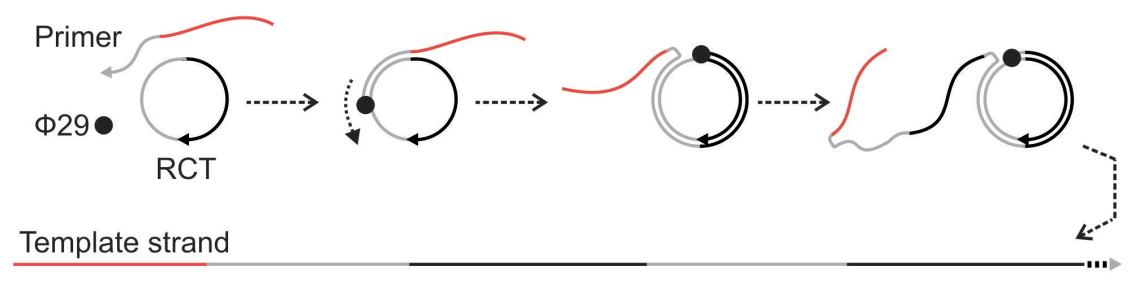

B DX tiles and spacer
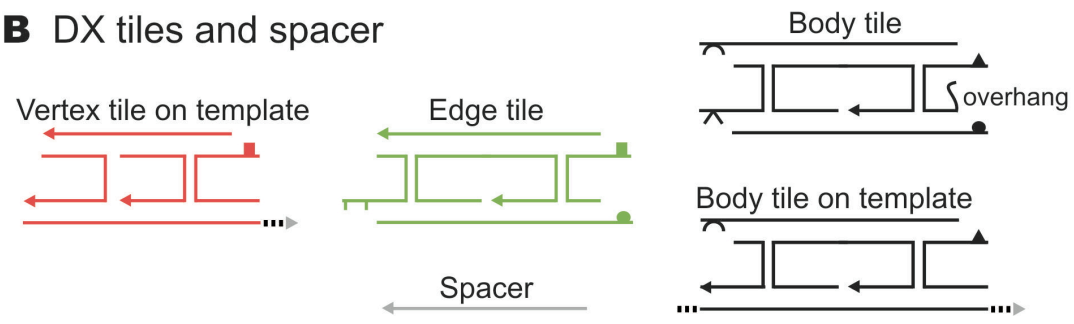

Figure 1. Tiles for self-assembled wedge-shaped arrays. A) Rolling circle replication reaction for synthesis of the array template strand. The phi29 polymerase, drawn as black dot, moves around the rolling circle template adding bases to the $3^{\prime}$ end of the primer (indicated by an arrow head) to create the periodic array template. A unique sequence motif carried by the primer is incorporated at the $5^{\prime}$ end (red). B) Three DX-tiles and a spacer strand form the remaining building blocks. The six sticky ends that connect tiles are indicated by lock and key symbols: square, semicircle and triangle. The vertex tile (red) can only assemble on the template. It has one sticky end. Edge tiles (green) assemble independently. They have three sticky ends. Body tiles (black) may assemble independently, in which case they have four sticky ends, or on the template, in which case they have two. The spacer strand (grey) hybridizes to the template strand between body tile sites. 


\section{Rolling circle replication}

\author{
Rolling circle template \\ Bracing strand \\ Rolling circle primer
}

\section{Building blocks of the array}

Array template $(n \approx 10)$

Vertex tile (on template)

Edge tile

Body tile (free)

(overhang in bold letters)

Body tile on template

Spacer
PhoGTTAAGTATAAGATGGCGAAAGCAGTGTCTATTGAGCCTACGTCCAGGTATGTGGTGATTAGTTGTTTAGGATGATAGATGAAG>

CCATCTTATACTTAACCTTCATCTATCATCC>

GCACTATCACTATCAGGCCGCAGCAGGACAGCAGCTTATCATAATCGAGTTCCTTCATCTATCATCCTAAAC>

GCACTATCACTATCAGGCCGCAGCAGGACAGCAGCTTATCATAATCGAGTTC-
(CTTCATCTATCATCCTAAACAACTAATCACCACATACCTGGACGTAGGCTCAATAGACACTGCTTTCGCCATCTTATACTTAAC) n $>$

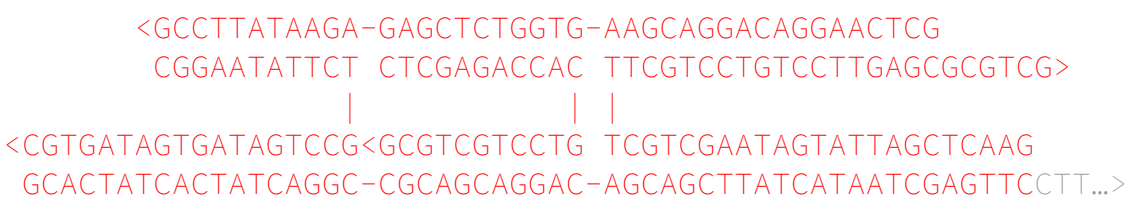
GCACTATCACTATCAGGC-CGCAGCAGGAC-AGCAGCTTATCATAATCGAGTTCCTT...>

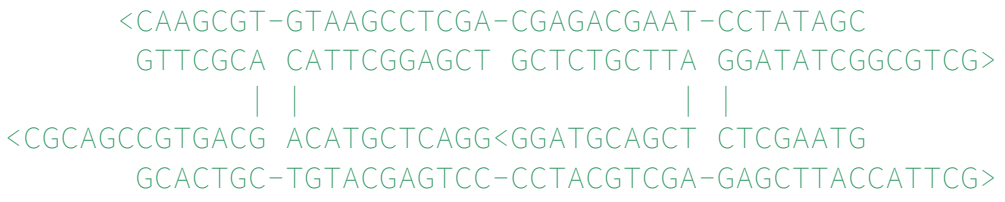

<CAAGCGT-GTAAGCCTCGA-CGAGACGAAT-CCTATAGC

GTTCGCA CATTCGGAGCT GCTCTGCTTA GGATATCGGCGTCG>

$<C G C A G C C G T G A C G$ ACATGCTCAGG $\angle G G A T G C A G C T$ CTCGAATG

GCACTGC-TGTACGAGTCC-CCTACGTCGA-GAGCTTACCATTCG>

<GTAAGCCGGAACG-GAGGGAAACCAT-TGACTACTG-CTTAGAGG

GCCTTGC CTCCCTTTGGTA-ACTGATGAC GAATCTCCCTGGAC>

|

$<$ GACCTGCATCCGA GTTATCTGTGAC<GAAAGCGGT AGAATATG ${ }^{\prime}$

GTAGGCT-CAATAGACACTG-CTTTCGCCA-TCTTATACCATTCG>

$<$ GTAAGCCGGAACG-GAGGGAAACCAT-TGACTACTG-CTTAGAGG

GCCTTGC CTCCCTTTGGTA-ACTGATGAC GAATCTCCCTGGAC>

$\mid$

|

$\angle$ GACCTGCATCCGA GTTATCTGTGAC $\angle$ GAAAGCGGT AGAATATGAATTG

...TACCTGGACGTAGGCT-CAATAGACACTG-CTTTCGCCA-TCTTATACTTAACCTT ... >

Table 1. DNA sequence design. Font colours match those used in Figures 1 and 2. Lines connect consecutive nucleotides. Arrowheads indicate the $3^{\prime}$ end of each strand.

The building blocks of the array are shown in Figure 1 and the nucleotide sequences of the component stands are given in Table 1. Body tiles, which make up most of the area of the array, have a double cross-over (DX) structure ${ }^{27}$ of type DAE-E: ${ }^{3}$ they consist of two double helices connected by strand exchange at two points. Each body tile has two pairs of complementary single-stranded 'sticky ends' (indicated by lock and key symbols in Figure 1B) arranged such that tiles linked by sticky-end hybridization tessellate to form a two-dimensional array. ${ }^{3}$ Body tiles can also assemble directly on the template to create a one-dimensional tile array. Direct assembly on 
the template is encouraged by the inclusion of a 5-nt overhang domain (Figure 1B) that is singlestranded in the free tile but forms additional base pairs when the template strand replaces the bottom strand of the tile. Spacer strands hybridize to the array template between body tiles. A single vertex tile binds to the unique sequence at the $5^{\prime}$ end of the template to complete this linear structure which forms the seed for cooperative growth of the two-dimensional array. Two types of tile assemble on the seed: free body tiles and edge tiles. Edge tiles are similar to body tiles but offer a different combination of sticky ends.

The melting temperature of an isolated pair of complementary sticky ends is in the range $0 \sim 10{ }^{\circ} \mathrm{C}$ under conditions used in our experiments. ${ }^{28}$ The interactions between the five strands that make up a tile are far stronger. When the system is heated above the melting temperature of all complexes, then cooled, the sequence of assembly is controlled by this hierarchy of interaction strengths: individual tiles, including tiles bound to the array template, form first, then free tiles assemble by sticky-end cohesion to form the array. Array formation is seeded by the template. In the absence of template, there is a significant activation barrier associated with the formation of a crystallization nucleus large enough that accreting tiles can be held by cooperative binding of at least two sticky ends. ${ }^{19}$

The designed tile assembly sequence is shown in Figure 2. Assembly temperatures given below correspond to experimental conditions and are estimated melting temperatures based on the nearest-neighbor model for DNA hybridization:28 they are consistent with ultraviolet absorption measurements used to monitor array assembly. Spacer strands hybridize to the array template strand at approximately $67^{\circ} \mathrm{C}$. Vertex and body tiles form on the array template strand at approximately $60^{\circ} \mathrm{C}$ completing the formation of the one-dimensional template. Figure 2-1 shows this designed first stage of assembly, which produces a one-dimensional array of body tiles assembled along the template with a vertex tile at one end. Free body and edge tiles also form at around $60^{\circ} \mathrm{C}$. Free body tiles have four sticky ends, edge tiles have three. Between each pair of tiles bound to the array template is a gap of the right size and flanked by two sticky ends of the right 

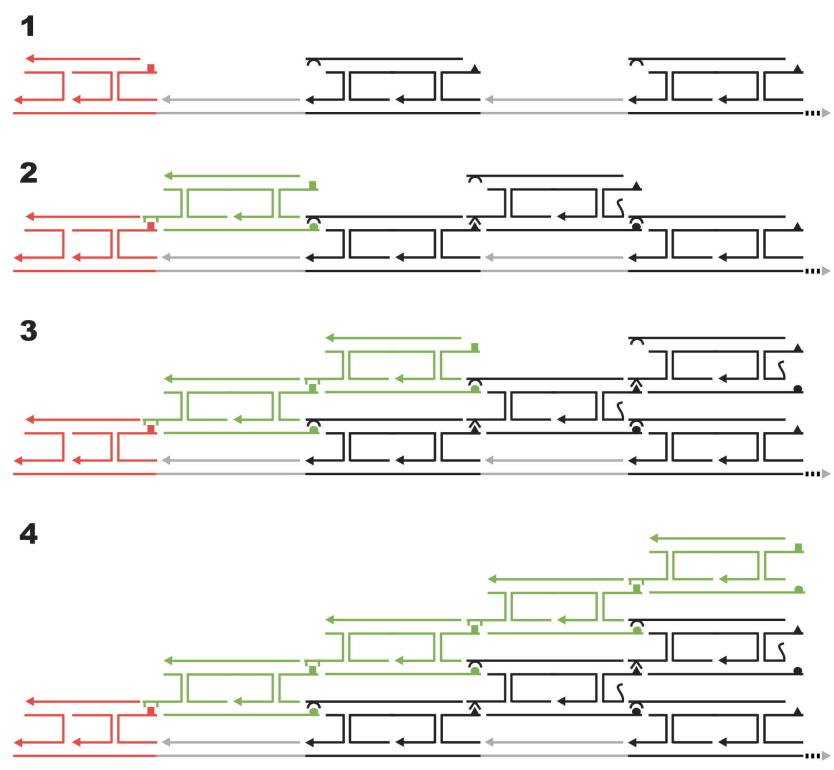

Figure 2. Assembly of the wedge-shaped array. 1) The one-dimensional seed formed by assembly of one vertex tile and a periodic array of body tiles and spacer strands on the array template. 2) to 4) Layer-by-layer self-assembly on the template: each incoming tile is attached by the formation of two bonds by hybridization of sticky ends.

type to bind, in the next row, a body tile or (next to the vertex tile) an edge tile (Figure 2-2). The special vertex and edge tiles form a stable border to the growing array: their limited valence defines its triangular shape. Row-by-row array assembly on the template occurs at around $45^{\circ} \mathrm{C}$ and is limited only by the length of the template and supply of tiles.

\section{Results}

Atomic force microscope (AFM) images of the three different assembly reactions are shown in Figure 3. Figure 3A shows that, in the absence of edge tiles or template, body tiles assemble to form narrow linear arrays with uniform width in the range $20 \sim 40 \mathrm{~nm}$, a measured height of approximately $2 \mathrm{~nm}$ and lengths of several micrometres. Note that the longest strand in this reaction has only 42 nucleotides, corresponding to a double helix of length approx. $13.6 \mathrm{~nm}$. The persistence length of these arrays is of the order of $10 \mu \mathrm{m}$. Figure $3 \mathrm{~B}$ shows that the first row of body tiles and the spacer strands can assemble on the periodic template as expected (Figure 2-1). 

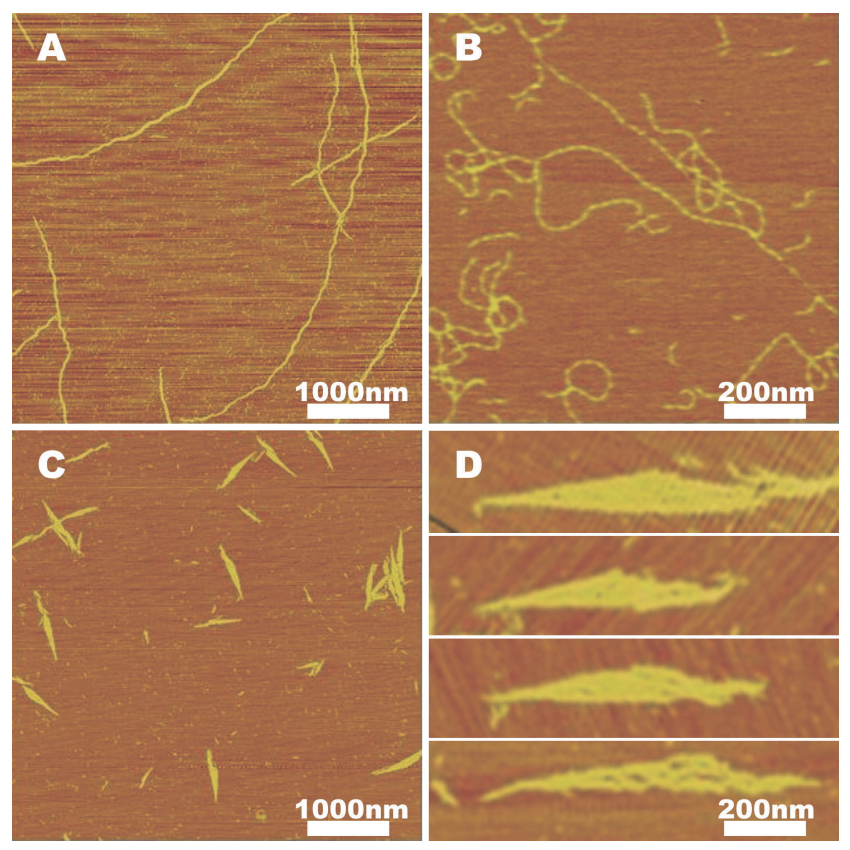

Figure 3. AFM images of self-assembly. A) Untemplated assembly of body tiles produces long, stiff tubes. B) One-dimensional templates assembled from template strand, spacer strands and body tiles (ex bottom strand). C) Products of templated self-assembly: triangular arrays form as designed. D) Magnified images of arrays.

The measured distance between template-bound tiles is $29 \pm 1 \mathrm{~nm}$, consistent with the expected period of $84 \mathrm{bp}(\sim 29 \mathrm{~nm})$. The height of the DX segments is approximately $1 \mathrm{~nm}$. The persistence length of these one-dimensional arrays is or the order of $100 \mathrm{~nm}$. Figure 3C shows the products of annealing all tiles. A number of two-dimensional arrays, which are quite uniform in size, are visible: four are shown at higher magnification in Figure 3D. From this and other images we estimate that approximately half of the assemblies have the designed triangular shape with a well-defined vertex angle of $12 \pm 2^{\circ}$. The shapes of other assemblies are also consistent with templated array growth but their vertex angles cannot be measured reliably. The height of the arrays is approximately $1 \mathrm{~nm}$.

\section{Discussion}

The stiff, linear arrays formed by assembly of DX body tiles in the absence of the template (Figure 3 A) are tubes. ${ }^{29}, 30$ The measured height is twice that of a single DX tile because the tubes are flattened onto the mica substrate during sample preparation. Seamless tubes, which may have a 
helical structure, avoid unsatisfied 'sticky end' bonds except at their open ends, reducing their energy relative to finite two-dimensional arrays. Tube formation may be encouraged by an inbuilt twist within each tile or along the helices joining tiles: body tiles are designed to assemble in the same orientation as their neighbors, so an intrinsic twist would cause a two-dimensional assembly of tiles to curl.

The persistence length of the one-dimensional array of body tiles and spacer strands assembled on the template strand (Figure 3B) is larger than, but of the same order of magnitude as, that of double-stranded DNA (50 nm), as expected. ${ }^{31}$ Body tile sites along the template are fully occupied.

The presence of the extended template strand to nucleate assembly, and edge tiles to control shape, completely changes the product of assembly, as designed (Figure 3C). The two edges of the arrays that are defined by the template and edge tiles are typically straight. The measured angle of $12 \pm 2^{\circ}$ is consistent with the expected value of $12.4^{\circ}$, which is based on a typical measured DX tile width of $6 \mathrm{~nm} \cdot .^{30}$ Arrays imaged contain of the order of 200 tiles each: this size is consistent with the stoichiometry of body and edge tiles and template binding sites.

DNA structures may be made conductive by metallization., 52-35 The tip of one of our arrays is an easily-functionalized double helix ( $2 \mathrm{~nm}$ diameter). The narrow vertex angle and micrometre length scale of the arrays suggest their application as adaptors between lithographically fabricated contacts and molecular-scale electronic devices. Another possible application is as a template on which to assemble (along the edges) two converging tracks for synthetic molecular motors.,36

\section{Conclusion}

We have fabricated DNA arrays with a controlled wedge shape using an extended onedimensional template and a minimal set of tiles. Observed array sizes are of the order of $1 \mu \mathrm{m}$; larger structures may be achievable by changing the ratio between tile types. These arrays may be suitable as templates for contacts to molecular electronic devices or for motor tracks. 


\section{Experimental Section}

DNA sequences were designed with the program NANEV which employs an evolutionary algorithm to optimize DNA design by minimizing unwanted complementarities of unrelated basesequences. ${ }^{37}$ Table 1 lists the DNA sequences for the strands used to create the template for rolling circle replication, the array template strand (product of the rolling circle replication), the tiles and the spacer strand. All enzymes were purchased from New England Biolabs (Ipswich, MA, USA). DNA oligonucleotides were purchased from IDT (Coralville, IA, USA). Concentrations were deduced from measurements of UV absorption at $260 \mathrm{~nm}$.

The 84-nt single-stranded rolling circle substrate was synthesized by ligating a 5' phosphorylated strand into a circle with the help of a complementary bracing strand using T4 DNA ligase in the buffer provided. Concentrations of component strands were adjusted to $1 \mu \mathrm{M}$. The ligation reaction was allowed to proceed for 30 minutes at $16^{\circ} \mathrm{C}$ and then stopped by heat inactivation. All non-circular DNA, including the bracing strand, was digested with Exol and Exolll in ligase buffer. Rolling circle replication was carried out with phi29 polymerase in the buffer provided using the 72-nt primer of which 20 nt at the 3' end were complementary to the RCT. The concentrations of RCT and primer were $0.5 \mu \mathrm{M}$. Rolling circle replication was allowed to proceed for 2 hours at $30^{\circ} \mathrm{C}$ then stopped by heat inactivation. Reaction products were purified by phenol-chloroform extraction. Alkaline agarose gel electrophoresis was used to separate the circular RCT from the linear template strand. The concentration of template repeat units was measured by titration against the spacer strand using polyacrylamide gel electrophoresis to separate and measure the concentration of surplus spacer strands.

Three annealing reactions with different components were prepared. The first contained only body tile components. The second contained array template strand, spacer strand and the four strands necessary to assemble body tiles on the template. The third contained all components. All reactions were prepared in $10 \mathrm{mM} \mathrm{MgCl}, 20 \mathrm{mM}$ Tris $\bullet \mathrm{HCl}$ and $1 \mathrm{mM}$ EDTA at $\mathrm{pH}$ 8.0. The concentration of repeat units on the template strands was $10 \mathrm{nM}$. The concentration of body tile strands was 200 nM; the concentrations of vertex tile strands, edge tile strands and spacer strand 
were $1.5 \mathrm{nM}, 20 \mathrm{nM}$ and $20 \mathrm{nM}$ respectively. The reaction mixtures were heated to $90^{\circ} \mathrm{C}$ then cooled to room temperature in a beaker of water in an expanded polystyrene box over a period of 36 hours. $20 \mu \mathrm{l}$ of each reaction was deposited on mica. After 10 minutes the mica was dried with nitrogen gas, washed with de-ionized water and then dried again. The samples were imaged in tapping mode in air using a Nanoman AFM (Veeco, Woodbury, NY, USA).

\section{References}

1. Seeman, N. C. Nature 2003, 421, 427-431.

2. Bath, J.; Turberfield, A. J. Nat. Nanotech. 2007, 2, 275-284.

3. Winfree, E.; Liu, F.R.; Wenzler, L. A.; Seeman, N. C. Nature 1998, 394, 539-544.

4. LaBean, T. H.; Yan, H; Kopatsch, J.; Liu, F. R.; Winfree, E.; Reif, J. H.; Seeman, N. C. J. Am. Chem. Soc. 2000, 122, 18481860.

5. Yan, H.; Park, S. H.; Finkelstein, G.; Reif, J. H.; LaBean, T. H. Science 2003, 301, 1882-1884.

6. Malo, J.; Mitchell, J. C.; Venien-Bryan, C.; Harris, J. R.; Wille, H.; Sherratt, D. J.; Turberfield, A. J. Angew. Chem. Int. Ed. $2005,44,3057-3061$.

7. He, Y.; Tian, Y.; Chen, Y.; Deng, Z. X.; Ribbe, A. E.; Mao, C. D. Angew. Chem. Int. Ed. 2005, 44, 6694-6696.

8. Lund, K.; Liu, Y.; Lindsay, S.; Yan, H. J. Am. Chem. Soc. 2005, 127, 17606-17607.

9. Liu, Y.; Ke, Y. G.; Yan, H. J. Am. Chem. Soc. 2005, 127, 17140-17141.

10. Park, S. H.; Pistol, C. Ahn, S. J.; Reif, J. H.; Lebeck, A. R.; Dwyer, C.; LaBean, T. H. Angew. Chem. Int. Ed. 2006, 45, 735739.

11. Park, S. H.; Finkelstein, G; LaBean, T. H. J. Am. Chem. Soc. 2008, 130, 40-41.

12. Winfree E. In DNA Based Computers; Lipton, R. J., Baum E. B., Eds.; American Mathematical Society: Providence, Rhode Island, 1996; Vol. 27, pp 199-221.

13. Rothemund, P. W. K.; Papadakis, N.; Winfree, E. PLoS Biology 2004, 2, 2041-2053.

14. Mao, C. D.; LaBean, T. H.; Reif, J.H.; Seeman, N. C. Nature 2000, 407, 493-496.

15. Barish, R. D.; Rothemund, P. W. K.; Winfree, E. Nano Lett. 2005, 5, 2586-2592.

16. Soloveichik, D.; Winfree, E. SIAM J. Comput. 2006, 36, 1544-1569.

17. Schulman, R.; Lee, S.; Papadakis, N.; Winfree, E. Lect. Notes Comput. Sc. 2004, 2943, 108-125.

18. Yan, H.; LaBean, T. H.; Feng, L. P.; Reif, J. H. Proc. Natl Acad. Sci. USA, 2003, 100, 8103-8108.

19. Schulman, R.; Winfree, E. Proc Natl Acad Sci USA, 2007, 104, 15236-15241.

20. Fire, A.; Xu, S. Q. Proc. Natl Acad. Sci. USA 1995, 92, 4641-4645.

21. Daubendiek, S. L.; Ryan, K; Kool, E. T. J. Am. Chem. Soc. 1995, 117, 7818-7819.

22. Lizardi, P. M.; Huang, X. H.; Zhu, Z. R. Bray-Ward, P.; Thomas, D. C.; Ward, D. C. Nat. Genet. 1998, 19, 225-232.

23. Blanco, L.; Bernad, A.; Lazaro, J. M.; Martin, G.; Garmendia, C.; Salas, M. J. Biol. Chem. 1989, 264, 8935-8940. 
24. Beyer, S.; Nickels, P.; Simmel, F. C. Nano Lett. 2005, 5, 719-722.

25. Deng, Z. X.; Tian, Y.; Lee, S. H.; Ribbe, A. E.; Mao, C. D. Angew. Chem. Int. Ed. 2005, 44, 3582-3585.

26. Lubrich, D.; Bath, J.; Turberfield, A. J. Nanotechnology, 2005, 16, 1574-1577.

27. Fu, T. J.; Seeman, N. C. Biochemistry, 1993, 32, 3211-3220.

28. SantaLucia, J., Jr. Proc. Natl Acad. Sci. USA, 1998, 95, 1460-1465.

29. Mitchell, J. C.; Harris, J. R.; Malo, J; Bath, J.; Turberfield, A. J. J. Am. Chem. Soc. 2004, 126, 16342-16343.

30. Rothemund, P. W. K.; Ekani-Nkodo, A.; Papadakis, N.; Kumar, A.; Fygenson, D. K.; Winfree, E. J. Am. Chem. Soc. 2004, $126,16344-16352$.

31. Sa-Ardyen, P.; Vologodskii, A. V.; Seeman, N. C. Biophys. J. 2003, 84, 3829-3837.

32. Braun, E.; Eichen, Y.; Sivan, U.; Ben-Yoseph, G. Nature 1998, 391, 775-778.

33. Richter, J.; Mertig, M.; Pompe, W.; Monch, I.; Schackert, H. K. Appl. Phys. Lett. 2001, 78, 536-538.

34. Keren, K.; Krueger, M.; Gilad, R.; Ben-Yoseph, G.; Sivan, U.; Braun, E. Science, 2002, 297, 72-75.

35. Liu, D.; Park, S. H.; Reif, J. H.; LaBean, T. H. Proc. Natl Acad. Sci. USA 2004, 101, 717-722.

36. Balzani, V.; Credi, A.; Raymo, F. M.; Stoddart, J. F. Angew. Chem. Int. Ed. 2000, 39, 3349-3391.

37. Goodman, R. P. Biotechniques, 2005, 38, 548-552. 